\title{
Spectral Analysis Assessment of Respiratory Sinus Arrhythmia in Normal Infants and Infants Who Subsequently Died of Sudden Infant Death Syndrome
}

\author{
KAREN A. KLUGE, RONALD M. HARPER, VICKI L. SCHECHTMAN, ADRIAN J. WILSON, \\ HOWARD J. HOFFMAN, AND DAVID P. SOUTHALL \\ Brain Research Institute, University of California at Los Angeles, Los Angeles, California, USA [K.A.K., R.M.H., \\ V.L.S.j; Department of Medical Physics and Clinical Engineering, Royal Hallamshire Hospital, Sheffield, \\ England [A.J.W.]; Biometry Branch, National Institute of Child Health and Human Development, National \\ Institutes of Health, Bethesda, Maryland [H.J.H.]; and Cardiothoracic Institute, Brompton Hospital,
} London, England [D.P.S.]

\begin{abstract}
Reduced heart rate variability has been found in infants who later succumb to the sudden infant death syndrome (SIDS). To determine whether respiratory sinus arrhythmia, a major component of heart rate variability, is also reduced in SIDS victims, nighttime portions of eighteen 24-h recordings of ECG and respiration from infants who later died of SIDS and 52 recordings from control infants were assessed using spectral analysis. Two aspects of respiratory sinus arrhythmia were examined: "extent" (the absolute heart rate variation at the respiratory frequency) and "coherence" (the degree to which heart rate follows respiration regardless of the absolute amount of variation). Respiratory parameters were used to classify each 1-min epoch as quiet sleep, rapid eye movement sleep, waking, or indeterminate state. Median extent and coherence values across the night were then computed for each sleep-waking state. Two-way (group $\times$ state) repeated measures analysis of variance tests were then used to compare respiratory sinus arrhythmia values for 13 SIDS victims and 13 control infants matched by postnatal age, birth weight, sex, and gestational age. Extent of respiratory sinus arrhythmia was significantly lower in the SIDS victims across all sleep-waking states, a finding that persisted after adjusting for heart rate. Coherence values did not differ significantly. These results suggest that even before the time of maximal risk for the syndrome, SIDS victims, as a group, differ from controls in the extent to which cardiac and respiratory activity couple, and this difference is independent of basal heart rate. (Pediatr Res 24: 677682, 1988)
\end{abstract}

Abbreviations

SIDS, sudden infant death syndrome RSA, respiratory sinus arrhythmia

Received October 14, 1987; accepted July 28, 1988.

Correspondence and reprint requests Karen A. Kluge, Brain Research Institute, UCLA, Los Angeles, CA 90024-1761.

Supported by Contract N01-HD-3-2830 and Grants HD 22695 and HD 22506 from the National Institute of Child Health and Human Development, United States Public Health Service (K.A.K., R.M.H., V.L.S.). Also supported by the National Institute of Mental Health and the National Sudden Infant Death Syndrome Foundation (V.L.S.), Nellcor Corporation (D.P.S.), and FSID (AJW).
REM, rapid eye movement

ANOVA, analysis of variance

A number of studies of the SIDS have investigated aspects of cardiac and respiratory activity, postulating that abnormal cardiorespiratory patterns may characterize risk for SIDS (1-10). Varying conclusions have been drawn regarding the degree of cardiorespiratory dysfunction in SIDS, depending in part on the risk population studied (siblings of SIDS victims, "near-miss for SIDS" infants, symptomatic infants who subsequently died suddenly, or asymptomatic infants who subsequently died of SIDS), the control population used for comparative purposes (asymptomatic infants versus "high-risk" infants who did not die), the data selection used (selected epochs of minimal variability or regular breathing versus all nonartifactual minutes over a longterm recording), and the hypotheses being tested (group difference between SIDS and controls versus predictive marker). For the most part these investigations have not taken into account the profound effect of sleep-waking state on cardiorespiratory measures because the recordings available for study usually do not include EEG, electromyogram, and electrooculogram, the parameters traditionally used to classify state. We developed a computer-based procedure that classifies infant sleep-waking state based on cardiorespiratory data alone (11), and have applied this algorithm to data from the prospective study of Southall et al. (12). We reported that when the data are partitioned by state and age, SIDS victims show higher heart rates in all states before 1 month of age, higher heart rates in REM sleep from 1-2 months of age, lower heart rate variability in waking before 1 month of age, and no difference in respiratory rate or variability in any state or age group (10).

The purpose of the present study was to examine a component of heart rate variability, RSA, the respiratory-related modulation of heart rate that is manifested as an increase in heart rate during inspiration and a decrease during expiration. Diminution or absence of RSA has been documented in asphyxiated infants, in severe cardiac disorders, in diabetic autonomic neuropathy, and in a number of other disorders (13-15). In an unpublished study, we noted that the amplitude of RSA was reduced in siblings of SIDS victims during quiet sleep at $1 \mathrm{wk}$ of age, and we wished to extend this investigation to infants who actually succumbed to SIDS. The present study therefore compared RSA in asymptomatic infants who subsequently became victims of SIDS and control infants, using the machine classification algorithm of 
Harper et al. (11) to partition the effect of sleep state, and spectral analysis procedures to assess RSA.

\section{MATERIALS AND METHODS}

Subject population. Twenty-four-hour recordings of cardiorespiratory data were acquired from 6914 full-term infants born in three maternity hospitals in England during the period July 1980 to July 1981 (12). Two recordings were made on most infants, one in the hospital during the first week of life (avoiding the first $24 \mathrm{~h}$ after birth) and one at home at approximately $6 \mathrm{wk}$ of age. In some cases, a single recording was obtained at home between 3 and 6 wk of age. Sixteen of the infants recorded subsequently died with a postmortem diagnosis of SIDS. Six of these infants were recorded twice, providing 22 recordings of infants who subsequently became victims of SIDS. From the remaining recordings, 66 were selected as being closely matched to the SIDS recordings in terms of age at the time of recording, gestational age, sex, and birth weight. These 88 recordings were provided to the Los Angeles investigators (K.A.K., V.L.S., and R.M.H.) by the British researchers (A.J.W. and D.P.S.).

In the course of conducting analyses of this data set (10), we encountered recordings we could not analyze, primarily because the number of artifacts in the cardiac signal made our automated sleep state classification procedures (see below) untenable. Any recording in which our automated artifact detection and correction program (16) identified more than $30 \%$ of the 1 -min epochs as containing uncorrectable artifacts in the cardiac R-R interval data was eliminated from analysis. A total of 18 of the 88 recordings were therefore excluded from analysis, four SIDS recordings and 14 control recordings. All the remaining 18 SIDS recordings (including duplicate recordings of five infants) and 52 control recordings (including three duplicates) were sleep staged and subjected to spectral analysis as described below. However, for purposes of statistical analysis, we eliminated one of each set of duplicate recordings and selected a single, best-matched control recording for each of the 13 remaining SIDS recordings, thereby providing the equal sample sizes necessary for a two-way (group $\times$ state) ANOVA. Selection of which of two duplicate recordings to use was neither random nor a consistent choice of the first or second recording; instead, we selected recordings so as to provide the best representation of the range of ages at time of recording (infants were recorded at 2-65 days of age, and we wished to have approximately equal numbers of infants above and below 1 month of age in our sample). Selection of the "bestmatched" control recording for each SIDS recording was made using the following criteria, applied in this order: 1) postnatal age \pm 1 day; ${ }^{1}$ 2) birth weight $\pm 300 \mathrm{~g}$; and 3) same sex. ${ }^{2}$ With three exceptions (noted in footnotes), all matched pairs met all three criteria. In cases where more than one infant met all the criteria, gestational age was also used as a matching variable, and infants who were most similar to the SIDS victims on all four matching criteria were selected for statistical analysis. All infants (SIDS victims and controls) had a gestational age of 37 or more wk and were considered to be full-term. The SIDS victims and control infants analyzed had comparable mean values for postnatal and gestational age and birth weight $(p>0.05$ in each case, $t$ tests), and comparable proportions of males to females ( $p>$ $0.05, \chi^{2}$; Table 1 ).

Signal processing. Cardiorespiratory data collected on each infant consisted of $24-\mathrm{h}$ recordings of ECG and respiratory waveform as assessed by detection of abdominal wall movement (17). Recordings of ECG and respiratory waveform, along with a $60-\mathrm{Hz}$ crystal clock signal to correct for tape speed variation, were made on a Medilog 4-channel recorder (Oxford Medical Systems, Abingdon, Oxon, England). The ECG, respiration, and

\footnotetext{
${ }^{1}$ In the case of one 2-month-old SIDS victim, a difference of 2 days was permitted in the matched control because the only control infant who met the \pm 1 day criterion weighed $930 \mathrm{~g}$ less than the SIDS victim and had a gestational age of $37 \mathrm{wk}$ (compared with $40 \mathrm{wk}$ ).

${ }^{2}$ Two exceptions were permitted where the only same-sex matches available would have violated the postnatal age and birth weight criteria.
}

Table 1. Comparison of matching variables in 13 SIDS victims and 13 matched control infants (mean $\pm S E$ ); no comparisons were significant at $\alpha=0.05$

\begin{tabular}{lcccc}
\hline & $\begin{array}{c}\text { Postnatal age } \\
\text { (days) }\end{array}$ & $\begin{array}{c}\text { Body wt } \\
(\mathrm{g})\end{array}$ & $\%$ Male & $\begin{array}{c}\text { Gestational } \\
\text { age (wk) }\end{array}$ \\
\hline Controls & $29 \pm 6.3$ & $3137 \pm 206$ & 62 & $39 \pm 0.3$ \\
SIDS & $29 \pm 6.4$ & $3119 \pm 225$ & 62 & $39 \pm 0.3$ \\
$p$ & 1.0 & 1.0 & 1.0 & 0.7 \\
\hline
\end{tabular}

$60-\mathrm{Hz}$ clock signals were transferred to $1 / 4$ inch reel-to-reel analog tapes and then digitized using a PDP $11 / 73$ computer. ECG signals were fed into a Schmitt trigger generator that produced a pulse for each $\mathrm{R}$ wave. Trigger event times were stored on digital tape along with the digitized analog signals. Cardiac interbeat intervals, calculated as the time between successive $\mathrm{R}$ waves, were computed to an accuracy of $2 \mathrm{~ms}$. A peak/trough algorithm was used to detect breaths from the digitized respiratory waveform, and breath-to-breath intervals were subsequently determined. Median breath-to-breath intervals and interquartile ranges were determined for nonoverlapping 1-min epochs throughout each recording to provide measures of respiratory rate and variability.

The R-R interval records were submitted to an automated artifact detection and correction program (16) that flagged as unusable 1-min epochs containing excessive artifacts. Epochs in which the median respiratory rate was excessively high or low ( $>110$ breaths/min or $<15$ breaths/min) were attributed to artifact and similarly eliminated from analysis. In addition, epochs containing large transient changes in the R-R interval values ${ }^{3}$ were eliminated from further analysis because such transients contain broadband spectral energy that would result in a poor estimate of the spectral composition of the signal. The number and percentage of minutes that were excluded from analysis ("artifactual" minutes) for the 13 pairs of SIDS victims and matched control infants analyzed are shown in Table 2.

Sleep state classification. We used a version of a recently developed automatic sleep state classification procedure (11) that requires only median respiratory rate and interquartile range to classify sleep state. This algorithm has an accuracy approximating that of two trained human observers using traditional sleep state scoring criteria (approximately $80 \%$ correct classification with respect to observer-assigned state codes). Because the discriminant function used to classify sleep state was determined from nighttime (1900-0700 h) recordings, only that portion of the prospective study data set was used. Each 1-min nighttime epoch was thereby coded as quiet sleep, REM sleep, waking, or indeterminate state.

Table 2 shows the number and percentage of epochs that were classified as each state for the 13 SIDS victims and 13 matched control infants analyzed. Epochs that were classified as indeterminate state ( $6 \%$ overall) were excluded from further analysis. A total of 14,271 quiet sleep, REM sleep, and waking minutes were therefore evaluated in the comparison of the 13 matched pairs of infants, an average of $549 \mathrm{~min} /$ infant.

We used a two-way (group $\times$ state), repeated measures ANOVA (BMDP P2V) on the number of minutes classified as each state to assess whether the SIDS victims and control infants had comparable sleep state organization. This ANOVA showed no group effect $[F(1,24)=2.18, p=0.15]$, but a significant group $\times$ state interaction $[F(4,96)=3.06, p=0.02]$. A Newman-Keuls' test on the interaction identified a significant difference in the number of artifactual minutes only (SIDS victims had significantly fewer artifactual minutes, $p<0.05$ ). We believe that this finding may be related to the reduced body movement reported for some infants at risk for SIDS (18-20), although not confirmed

${ }^{3}$ A "large transient change" was considered to be a sudden change in the slope of the R-R interval curve such that

$\left|\Delta R R_{(n-1)}\right|+\left|\Delta R R_{n}\right|>180$

where $\Delta R R=$ the difference between two subsequent $R-R$ interval values (16). 
Table 2. Number and \% of min classified as quiet sleep, REM, waking, and indeterminate state and min excluded from analysis because of excessive artifacts for the 13 SIDS victims and 13 matched control infants*

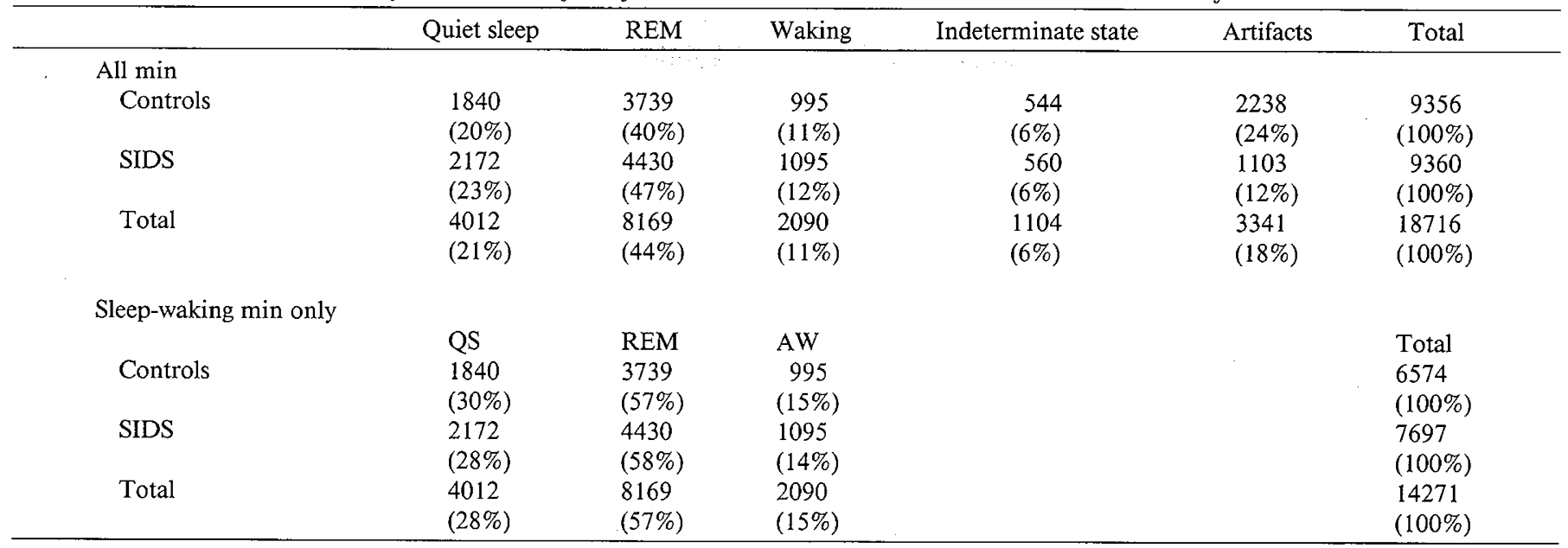

* When the artifacts and indeterminate state min were excluded, the SIDS victims and control infants had comparable percentages of quiet sleep, REM sleep, and waking minutes.

in other studies $(21,22)$, and are continuing to investigate this possibility.

Spectral analysis. Data input to the spectral analysis consisted of sampled curves of heart rate and respiratory waveform. The heart rate curve was constructed by linear interpolation between the reciprocals of successive $\mathrm{R}-\mathrm{R}$ intervals. The resulting function was sampled at 16 samples/s, as were the raw digitized respiratory values. Both heart rate and respiratory waveform curves were demeaned, cosine tapered to reduce leakage, and prewhitened at $\alpha=0.2$ to reduce the contribution of slow variation in highfrequency bands.

For each 1-min epoch of data, the heart rate curve and the respiratory waveform were each subjected to spectral analysis, yielding 512 raw autospectral bands. The cross-spectra of heart rate and respiration were also calculated for these 512 raw bands. For increased stability, the mean of each group of four consecutive spectral coefficients was then determined, thereby "collapsing" four raw bands to one and yielding an effective frequency resolution of $0.06 \mathrm{~Hz}$. We defined the peak in the respiratory autospectrum as the band in the range of 0.37 to $2.0 \mathrm{~Hz}$ (corresponding to respiratory rates between 22 and 120 breaths/min) with the greatest respiratory autospectral power. The heart rate autospectral and cross-spectral values for this band and one band on either side of it were used to compute RSA measures (Fig. 1). We used three bands (total width of $0.18 \mathrm{~Hz}$ ) to ensure that the total power of a fairly broad peak would be detected, even if the peak were asymmetric. Visual inspection of 400 spectral histograms indicated that these three bands included approximately $90 \%$ of the spectral energy of the respiratory peak in all sleepwaking states for both SIDS victims and controls.

Assessment of RSA. Two measures of RSA were used, as defined previously (23). The first measure, "extent of RSA," assesses the absolute amount of heart rate variability at the respiratory frequency. For each minute, extent of RSA was calculated as the log of the summed power of the heart rate autospectrum in the three bands surrounding the peak in the respiratory autospectrum. The second measure of RSA assesses the proportion of heart rate variability at these frequencies that is attributable to respiratory activity, independent of the absolute amount of variability; this measure is designated the "coherence of RSA." For each minute, coherence of RSA was calculated as the cross-spectral coherence between heart rate and respiratory activity in the three bands containing the peak in the respiratory autospectrum. Cross-spectral coherence values range from 0.0 to 1.0 and are analogous to squared correlation coefficients between two variables (24).
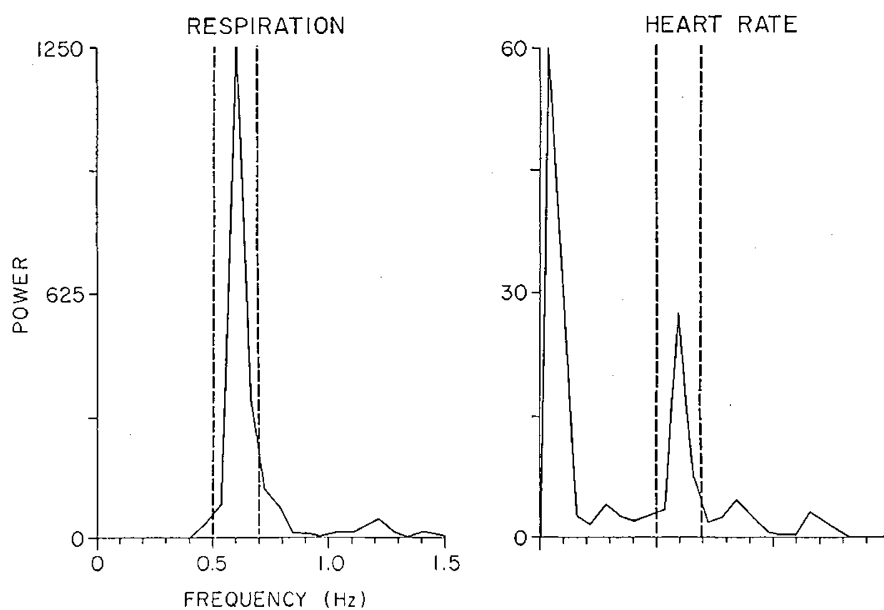

Fig. 1. Histograms of respiratory and heart rate autospectra for one quiet sleep minute from a 7-day-old infant. The peak in the respiratory autospectrum (three bands within dotted lines) was used to identify the RSA bands in the heart rate autospectrum (same three bands enclosed by dotted lines). Ordinate (power in arbitrary units) is shown as a linear scale; $\log$ power values were used in the calculation of extent and coherence measures.

From the minute by minute values of extent and coherence of RSA, we computed median values for each sleep state over the 12-h nighttime period for each infant. Median values were used instead of means to prevent distortion of the measure of central tendency by a few aberrant values. Two-way, repeated measures ANOVA tests (group $\times$ state; BMDP P2V) were then used to compare extent and coherence values of the 13 SIDS victims with those of the 13 control infants across sleep states. To determine if any observed differences were related to the increased heart rates seen in these SIDS victims (10), we assessed the correlation of each RSA measure with heart rate, and repeated the analyses after correcting for heart rate effects (vide infra).

\section{RESULTS}

Figures 2 and 3 present the median extent and coherence of RSA by state for the 13 SIDS victims and the 13 matched control infants used in the statistical analysis. Although the SIDS victims' values consistently fall within the range of the control infants' values, only one or two SIDS victims' values fall above the mean 


\section{QS}

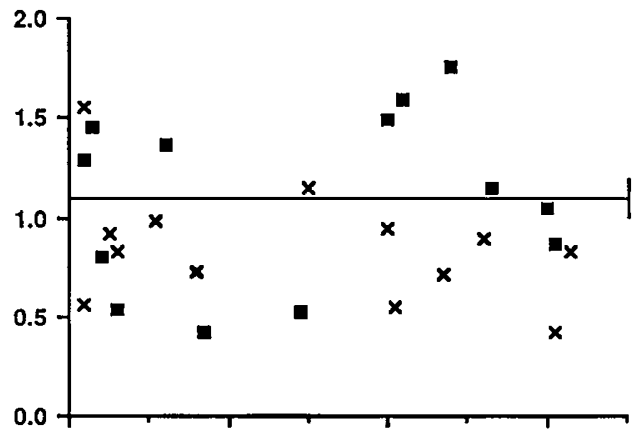

REM
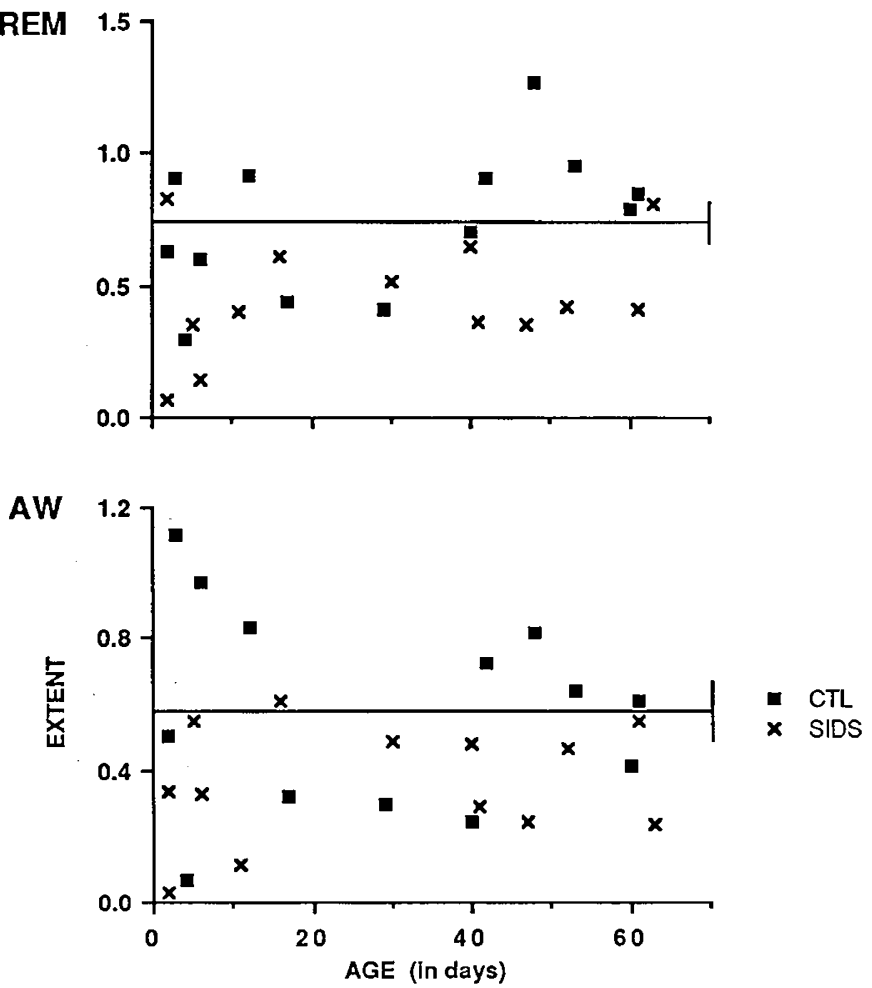

Fig. 2. Median extent of RSA (log of spectral power measured in arbitrary units) by age for the 13 SIDS victims (X) and 13 matched control infants (squares) analyzed in quiet sleep (QS), rapid eye movement sleep (REM), and waking (AW). Horizontal line and vertical bar indicate the mean $\pm S E$ of the 13 control infants' extent values. In each sleep state, all but one or two of the SIDS victims' values fall below the mean of the controls:

of the control subjects for extent of RSA in each sleep state. The scatter of coherence values is similar for the two groups in all sleep states.

Means and SE of extent (Fig. 4) and coherence (Fig. 5) of RSA by state are shown for the 13 SIDS victims and the 13 matched control infants studied. A two-way, repeated measures ANOVA test identified a reduction in the extent of RSA for the SIDS victims across all sleep-waking states [significant group effect, $F(1,24)=7.20, p=0.01$; no group $\times$ state interaction, $F(2,48)$ $=0.15, p=0.86]$. There was no significant difference between the two groups for the coherence measure $[F(1,24)=1.01, p=$ 0.33 .

Table 3 presents the correlation of median extent and coherence of RSA with median heart rate in each sleep-waking state for the 26 recordings studied. Because of the inequality of regression coefficients between the two groups, partitioning of heart rate effects could not be achieved by traditional analysis of covariance procedures. Instead, we corrected for heart rate by dividing each infant's median extent of RSA value for each state
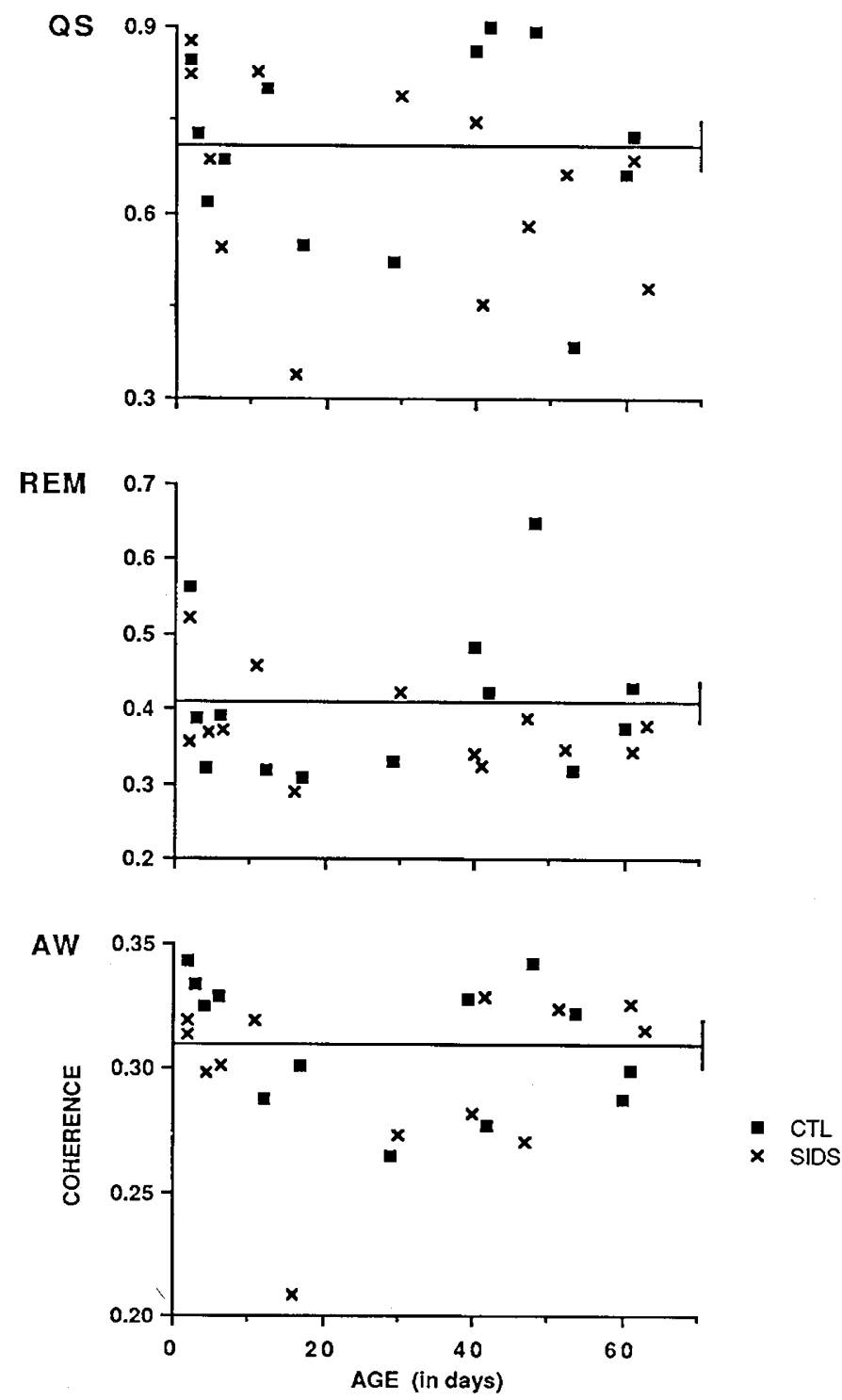

Fig. 3. Median coherence of RSA by age for the 13 SIDS victims and 13 matched control infants analyzed. Abbreviations and symbols as in Figure 2. The scatter of points for the two groups is similar.

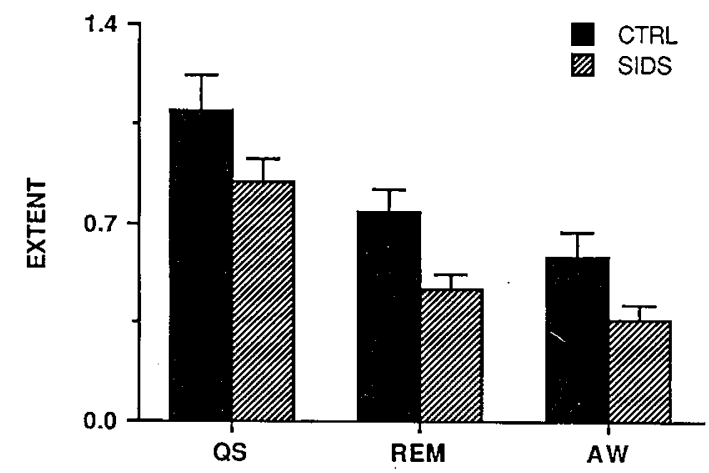

Fig. 4. Comparison of mean \pm SE extent of RSA in the 13 SIDS victims (hatched bars) and 13 matched control infants (solid bars) across sleep-waking states. Abbreviations as in Figure 2. Extent values of SIDS victims are significantly lower across all states (see text).

by the corresponding median heart rate squared $(6,25)$. The group effect for this ANOVA was still significant $[F(1,24)=6.10$, $p=0.02]$. No consistent relationship between coherence of RSA and heart rate was found in these data (Table 3) or in previous 


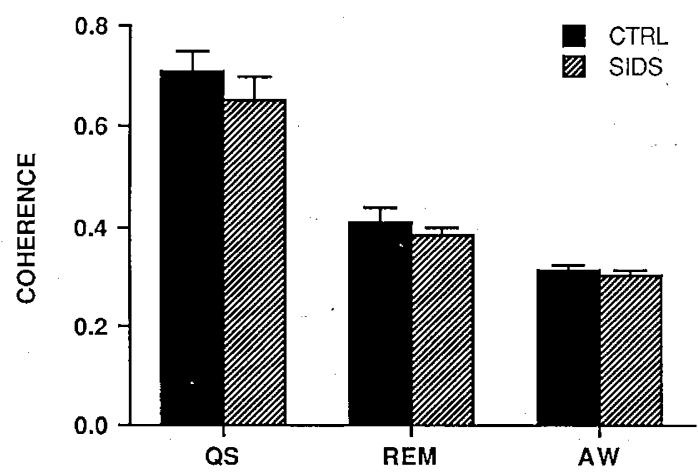

Fig. 5. Comparison of mean \pm SE coherence of RSA in the 13 SIDS victims (hatched bars) and 13 matched control infants (solid bars) across sleep-waking states. Abbreviations as in Figure 2. Coherence values of SIDS victims and controls are not significantly different (see text).

Table 3. Correlation of median extent and coherence of $R S A$ with median heart rate in each sleep-waking state for 13 SIDS victims and 13 best-matched controls

\begin{tabular}{llll}
\hline & & Pearson's $r$ & \multicolumn{1}{c}{$p$} \\
\hline Extent & & & $\ddots$ \\
Quiet sleep & Controls & -0.54 & 0.06 \\
& SIDS & -0.47 & 0.1 \\
& Combined & -0.53 & $0.004^{*}$ \\
REM sleep & Controls & -0.62 & $0.02^{*}$ \\
& SIDS & -0.20 & 0.5 \\
& Combined & -0.50 & $0.01^{*}$ \\
Waking & Controls & -0.44 & 0.1 \\
& SIDS & 0.15 & 0.6 \\
& Combined & -0.23 & 0.3 \\
Coherence & & & \\
Quiet sleep & Controls & -0.55 & $0.05^{*}$ \\
& SIDS & -0.12 & 0.7 \\
REM sleep & Combined & -0.35 & 0.1 \\
& Controls & -0.43 & 0.2 \\
& SIDS & -0.33 & 0.3 \\
Waking & Combined & -0.41 & $0.04^{*}$ \\
& Controls & 0.36 & 0.2 \\
& SIDS & 0.11 & 0.7 \\
& Combined & 0.10 & 0.6 \\
\hline
\end{tabular}

* Statistical significance at $\alpha=0.05$.

analyses of normal infants (23); therefore, we did not perform any correction of these values for heart rate effects.

\section{DISCUSSION}

These findings indicate that, as a group, infants who subsequently became victims of SIDS differ from control infants in having reduced extent of RSA. These findings cannot be interpreted to mean that low values on this variation measure are indicative of increased risk for SIDS, because the extent values of SIDS victims consistently fell within the range of control values. Nevertheless, extent values of SIDS victims, with few exceptions, fell below the mean of the controls' values, and the ANOVA demonstrates that this reduction is statistically significant across all sleep-waking states.

These findings therefore indicate that the difference in overall heart rate variability observed between control infants and infants who subsequently became victims of SIDS (10) may be further partitioned by source of variability. The difference observed was confined to the extent of RSA, rather than coherence. Respiratory and cardiac activity are therefore coupled in both groups, with similarly constant phase relationships. Because the difference in extent of RSA persists after correcting for heart rate effects, we conclude that this reduction in extent of RSA is independent of the heart rate difference previously reported (10).

Our previous investigation of SIDS siblings (Harper RM, Leake $B$, unpublished data) indicated a reduction in extent of RSA during quiet sleep, but only during the first week of life. Results of the present study demonstrate that in infants who become SIDS victims, reduced RSA persists at least into the second postnatal month and is found across all sleep-waking states.

Our finding of reduced extent of RSA in SIDS victims across all sleep states seems paradoxical in light of the previous finding of reduced overall heart rate variability (as measured by interquartile range) in SIDS victims in the waking state only (10). However, RSA is typically superimposed on larger, lower frequency variations (associated with blood pressure, thermoregulatory mechanisms, or body movement) that have a greater effect on interquartile range than does RSA. We are continuing to examine the contributions of lower frequency variation to overall heart rate variability, and are exploring measures of beat to beat variability that may be more appropriate to this investigation.

The application of a sleep state classification algorithm developed on healthy control infants to infants who may manifest pathologic physiology is a potential source of concern. We are beginning tests of the algorithm to ascertain how robust it is in pathologic cases. For purposes of the present study, it was necessary to partition by sleep state to account for the large intrasubject variability introduced by state, and this algorithm provided the only ready means of state scoring. The algorithm is unlikely to have influenced our findings because the difference in extent of RSA was seen across all sleep states, and the percentages of time spent in each state were comparable for SIDS victims and controls (after the artifactual minutes were eliminated).

Cardiac and respiratory activity in another group of infants who subsequently died suddenly was investigated by Gordon et al. (3). Indications for recording the eight sudden death victims studied were near-miss for SIDS events, choking spells, and/or the SIDS death of one or two siblings. These investigators confined their study to 256-s epochs in which there was regular respiration and minimal cardiac variability (probably quiet sleep, although no sleep staging was performed). These researchers found no difference in extent of RSA between the infants who died and the controls. The difference in our findings may be due to the use by Gordon et al. (3) of observer-selected epochs of minimal variability, compared with our analysis of all nonartifactual minutes over a 12-h period, as well as the major differences in the subject population. Gordon et al. (3) also reported a significantly increased dispersion of respiratory frequency (as assessed by manual calculation of respiratory autospectral bandwidth) in the victims. Enhanced respiratory rate variability has not been found in the SIDS victims studied here $(4,5,10)$, only one of whom had suffered cynotic episodes before the recording. (This subject was not included in the 13 cases subjected to statistical analysis.) Moreover, Gordon et al. (6) were unable to confirm their finding of increased respiratory bandwidth in a similar study of the present group of SIDS cases.

The dependence of RSA on respiratory rate and tidal volume has been noted by several investigators (26-28). An increase in respiratory rate or a reduction in tidal volume in SIDS victims could therefore account for the diminished RSA described here. No difference in respiratory rate has been reported for the group of SIDS victims studied here $(4-6,10)$. We were, of course, unable to determine tidal volume from infants instrumented only with an abdominal strain gauge; therefore, the diminished RSA values we reported for SIDS victims may result from reduced tidal volume, a costly but testable hypothesis.

Another factor that may contribute to reduced RSA is dysfunction of peripheral sensory receptors. Increased concentration of dopamine has been reported in the carotid bodies of SIDS victims (29). Inasmuch as chemoreflexes mediated by the carotid body affect cardiovagal tone differentially depending on respira- 
tory phase (30), anomalies of these chemosensitive transducers in infants at risk for SIDS may affect extent of RSA. Tne generality of the dopamine findings must be determined [particularly in light of the report by Lack et al. (31)]; the implications for SIDS physiology, if the findings can be generalized to most SIDS victims, are far-reaching.

Infants subjected to birth asphyxia exhibit diminished respiratory-related heart rate variation during quiet sleep (REM sleep and waking were not examined) (13). SIDS victims and infants subjected to asphyxia may share a common mechanism of reduced RSA. This possibility, however, provides little help in the identification of that mechanism, because asphyxia can interfere with a large number of central mechanisms, many of which could contribute to diminished vagal efferent activity associated with RSA. An effort should be directed toward examining the mechanisms contributing to diminished RSA in asphyxia and determining whether similar factors operate in potential SIDS victims.

Our results indicate that particular cardiac variability signs found in infants who succumb to SIDS are present very early in life, and support earlier reports of disturbed physiologic activity in infants at risk for SIDS well before the time of maximal risk for the syndrome (2-4 months). Inasmuch as the variability differences are present as early as the 1st wk of life, some disturbance of prenatal development seems likely, and the mechanisms that result in later failure may be detectable before developmental circumstances of maximal risk are attained. However, it should be reiterated that the results obtained in this study describe a group difference in respiratory-related heart rate variation. The findings should not be interpreted to mean that low values on this variation measure necessarily mean a risk for SIDS, because some control infants had similarly low values for particular states. Instead, the results should be used as a further impetus for examining particular aspects of autonomic function that might contribute to mechanisms of failure.

Acknowledgements. The data were provided by the British Heart Foundation, the Foundation for the Study of Infant Death (FSID), and the people of Brighton and Doncaster, England. We thank Dr. Robert Frysinger, Department of Anatomy, UCLA, for many constructive suggestions.

\section{REFERENCES}

1. Leistner HL, Haddad GG, Epstein RA, Lai TL, Epstein MAF, Mellins RB 1980 Heart rate and heart rate variability during sleep in aborted sudden infant death syndrome. J Pediatr 97:51-55

2. Harper RM, Leake B, Hodgman JE, Hoppenbrouwers T 1982 Developmental patterns of heart rate and heart rate variability during sleep and waking in normal infants and infants at risk for the sudden infant death syndrome. Sleep 5:28-38

3. Gordon D, Cohen RJ, Kelly D, Akselrod S, Shannon DC 1984 Sudden infant death syndrome: abnormalities in short term fluctuations in heart rate and respiratory activity. Pediatr Res 18:921-926

4. Wilson AJ, Stevens V, Franks CI, Alexander J, Southall DP 1985 Respiratory and heart rate patterns in infants destined to be victims of sudden infant death syndrome: average rates and their variability measured over 24 hours. Br Med J 290:497-501

5. Stevens V, Wilson AJ, Southall DP, Barber DC, Franks CI 1985 Analysis of the heart rate and breathing patterns of infants destined to suffer sudden infant death syndrome: probability density function analysis. Pediatr Res 19:1327-1332
6. Gordon D, Southall DP, Kelly DH, Wilson A, Akselrod S, Richards J, Kenet B, Kenet R, Cohen R, Shannon DC 1986 Analysis of heart rate and respiratory patterns in sudden infant death syndrome victims and control infants. Pediatr Res 20:680-684

7. Kelly DH, Golub H, Carley D, Shannon DC 1986 Pneumograms in infants who subsequently died of sudden infant death syndrome. J Pediatr 109:249254

8. Shannon DC, Kelly DH, Akselrod S, Kilborn KM 1987 Increased respiratory frequency and variability in high risk babies who die of sudden infant death syndrome. Pediatr Res 22:158-162

9. Southall DP, Stevens V, Franks CI, Newcombe RG, Shinebourne EA, Wilson AJ 1988 Sinus tachycardia in infants subsequently suffering from the sudden infant death syndrome. Eur J Pediatr 147:74-78

10. Schechtman VL, Harper RM, Kluge KA, Wilson AJ, Hoffman HJ, Southall DP 1988 Cardiac and respiratory patterns in normal infants and victims of the sudden infant death syndrome. Sleep 11:413-424

11. Harper RM, Schechtman VL, Kluge KA 1987 Machine classification of infant sleep state using cardiorespiratory measures. EEG Clin Neurophysiol 67:379387

12. Southall DP, Richards JM, de Swiet M, Arrowsmith WA, Cree JE, Fleming PJ, Franklin AJ, Orme RL'E, Radford MJ, Wilson AJ, Shannon DC, Alexander JR, Brown NJ, Shinebourne EA 1983 Identification of infants destined to die unexpectedly during infancy: evaluation of predictive importance of prolonged apnoea and disorders of cardiac rhythm or conduction. Br Med J 286:1092-1096

13. Divon MY, Winkler H, Yeh S-Y, Platt LD, Langer O, Merkatz IR 1986 Diminished respiratory sinus arrhythmia in asphyxiated term infants. Am J Obstet Gynecol 155:1263-1266

14. Grassman E, Blomqvist CG 1983 Absence of respiratory sinus arrhythmia: a manifestation of the sick sinus syndrome. Clin Cardiol 6:151-154

15. Mackay JD 1983 Respiratory sinus arrhythmia in diabetic neuropathy. Diabetologia 24:253-256

16. Schechtman VL, Kluge KA, Harper RM 1988 Time-domain system for assessing variation in heart rate. Med Biol Eng Comput 26:367-373

17. Wright M, Callan BM 1980 A new respiratory recording and monitoring system. In: Stott FD, Raftery EB, Goulding L (eds) Proceedings of the Third International Symposium on Ambulatory Monitoring. Academic Press, London, pp 329-334

18. Naeye RL, Messmer J III, Specht T, Merritt TA 1976 Sudden infant death syndrome temperament before death. J Pediatr 88:511-515

19. Hoppenbrouwers T, Jensen D, Hodgman J, Harper R, Sterman M 1982 Body movements during quiet sleep (QS) in subsequent siblings of SIDS. Clin Res 30:136A(abstr)

20. Coons S. Guilleminault C 1985 Motility and arousal in near miss sudden infant death syndrome. J Pediatr 107:728-732

21. Weissbluth M, Brouillette RT, Liu K, Hunt CE 1982 Sleep apnea, sleep duration, and infant temperament. J Pediatr 101:307-310

22. Vecchierini-Blineau M-F, Nogues B, Louvet S 1986 Etude des mouvements corporels au cours du sommeil chez le nourrisson: comparaison entre nourrissons temoins et nourrissons issus d'une fratrie de mort subite inexpliquee du nourrisson. Rev EEG Neurophysiol Clin 16:21-27

23. Harper RM, Walter DO, Leake B, Hoffman HJ, Sieck GC, Sterman MB Hoppenbrouwers T, Hodgman J 1978 Development of sinus arrhythmia during sleeping and waking states in normal infants. Sleep 1:33-48

24. Orr WC, Naitoh P 1976 The coherence spectrum: an extension of correlation analysis with applications to chronobiology. Int J Chronobiol 3:171-192

25. Mazza NM, Epstein MAF, Haddad GG, Law HS, Mellins RB, Epstein RA 1980 Relation of beat-to-beat variability to heart rate in normal sleeping infants. Pediatr Res 14:232-235

26. Angelone A, Coulter NA 1964 Respiratory sinus arrhythmia: a frequency dependent phenomenon. J Appl Physiol 19:479-482

27. Hirsch JA, Bishop B 1981 Respiratory sinus arrhythmia in humans: how breathing pattern modulates heart rate. Am J Physiol 24:H620-H629

28. Kitney RI 1984 New findings in the analysis of heart rate variability in infants. Automedica 5:289-310

29. Perrin DG, Cutz E, Becker LE, Bryan AC, Madapallimatum A, Sole MJ 1984 Sudden infant death syndrome: increased carotid-body dopamine and noradrenaline content. Lancet 2:535-537

30. Gandevia SC, McCloskey DI, Potter EK 1978 Inhibition of baroreceptor and chemoreceptor reflexes on heart rate by afferents from the lungs. $J$ Physiol (Lond) 276:369-381

31. Lack EE, Perez-Atayde AR, Young JB 1986 Carotid bodies in sudden infant death syndrome: a combined light microscopic, ultrastructural, and biochemical study. Pediatr Pathol 6:335-350 